Clinical Investigation

\title{
Neonatal autonomic function after pregnancy complications and early cardiovascular development
}

Christina Y. L. Aye ${ }^{1,2}$, Adam James Lewandowski ${ }^{1}$, Julien Oster ${ }^{3}$, Ross Upton ${ }^{1}$, Esther Davis ${ }^{1}$, Yvonne Kenworthy ${ }^{1}$, Henry Boardman ${ }^{1}$, Grace Z. Yu ${ }^{1}$, Timo Siepmann ${ }^{1}$, Satish Adwani ${ }^{4}$, Kenny McCormick ${ }^{4}$, Yrsa B. Sverrisdottir ${ }^{5}$ and Paul Leeson ${ }^{1}$

BACKGROUND: Heart rate variability (HRV) has emerged as a predictor of later cardiac risk. This study tested whether pregnancy complications that may have long-term offspring cardiac sequelae are associated with differences in HRV at birth, and whether these HRV differences identify abnormal cardiovascular development in the postnatal period.

METHODS: Ninety-eight sleeping neonates had 5-min electrocardiogram recordings at birth. Standard time and frequency domain parameters were calculated and related to cardiovascular measures at birth and 3 months of age.

RESULTS: Increasing prematurity, but not maternal hypertension or growth restriction, was associated with decreased HRV at birth, as demonstrated by a lower root mean square of the difference between adjacent NN intervals (rMSSD) and low (LF) and highfrequency power (HF), with decreasing gestational age $(p<0.001, p=0.009$ and $p=0.007$, respectively). We also demonstrated a relative imbalance between sympathetic and parasympathetic tone, compared to the term infants. However, differences in autonomic function did not predict cardiovascular measures at either time point.

CONCLUSIONS: Altered cardiac autonomic function at birth relates to prematurity rather than other pregnancy complications and does not predict cardiovascular developmental patterns during the first 3 months post birth. Long-term studies will be needed to understand the relevance to cardiovascular risk.

Pediatric Research (2018) 84:85-91; https://doi.org/10.1038/s41390-018-0021-0

\section{INTRODUCTION}

Heart rate variability (HRV) analysis provides a non-invasive measure of cardiac autonomic function, based on variation in the QRS to QRS (RR or normal to normal (NN) interval) interval sequence of the electrocardiogram (ECG). The derived metrics of HRV allow evaluation of sympathetic and parasympathetic balance within the autonomic nervous system (ANS) and the ability of the sinoatrial node to adapt to extrinsic signals. In a multitude of well-designed studies, decreased HRV has emerged as a strong predictor of cardiac risk in adults and death in patients at increased cardiovascular risk. $^{1-3}$ Interestingly, attenuation in HRV is also evident in infants born preterm, ${ }^{4}$ with dysfunction being greater in those with higher clinical illness scales ${ }^{5}$ or pathological problems such as respiratory distress syndrome, ${ }^{6}$ birth asphyxia, ${ }^{6}$ intraventricular haemorrhage ${ }^{6}$ and small for gestational age (SGA). ${ }^{7}$ Pregnancy complications, in particular, preterm birth and maternal hypertension have been found to be associated with an increased risk of cardiovascular disease in later life ${ }^{8}$ and the offspring display a distinct cardiovascular phenotype, characterised by microvascular rarefaction and cardiac hypertrophy. ${ }^{9-11}$

These cardiac and vascular patterns become evident during the first 3 months of life, when differences in autonomic function have been identified in preterm infants. ${ }^{10,12,13}$ Therefore, we investigated, for the first time, using short ECG recordings in a large cohort of newborn infants, whether differences in neonatal HRV relates just to prematurity or are found in other pregnancy complications linked with later cardiovascular disease. Furthermore, we studied whether altered HRV may be a marker of abnormal in utero or postnatal cardiac and vascular development in these infants.

\section{METHODS}

Study overview

Between 2011 and 2015, 600 mothers being cared for by the Oxford University Hospitals NHS Foundation Trust were identified by their clinical care team and invited to participate in one or more portfolio of studies coordinated by the Oxford Cardiovascular Clinical Research Facility. These studies were designed to investigate the impact of pregnancy complications on cardiovascular development during fetal and neonatal life, and used a stratified recruitment approach to ensure balanced representation of preterm and term birth, as well as hypertensive and normotensive pregnancies.

To study HRV, cardiac and microvascular development, we used the available ECG, echocardiographic and in vivo microvascular

\footnotetext{
${ }^{1}$ Oxford Cardiovascular Clinical Research Facility, Division of Cardiovascular Medicine, Radcliffe Department of Medicine, University of Oxford, Oxford, UK; ${ }^{2}$ Nuffield Department of Obstetrics \& Gynaecology, Medical Sciences Division, University of Oxford, Oxford, UK; ${ }^{3}$ Institute of Biomedical Engineering, University of Oxford, Oxford, UK; ${ }^{4}$ Department of Paediatrics and Neonatology, John Radcliffe Hospital, Oxford, UK and ${ }^{5}$ Nuffield Department of Clinical Neurosciences, University of Oxford, Oxford, UK Correspondence: Paul Leeson (paul.leeson@cardiov.ox.ac.uk)
}

Received: 1 December 2017 Revised: 8 February 2018 Accepted: 10 March 2018

Published online: 23 May 2018 


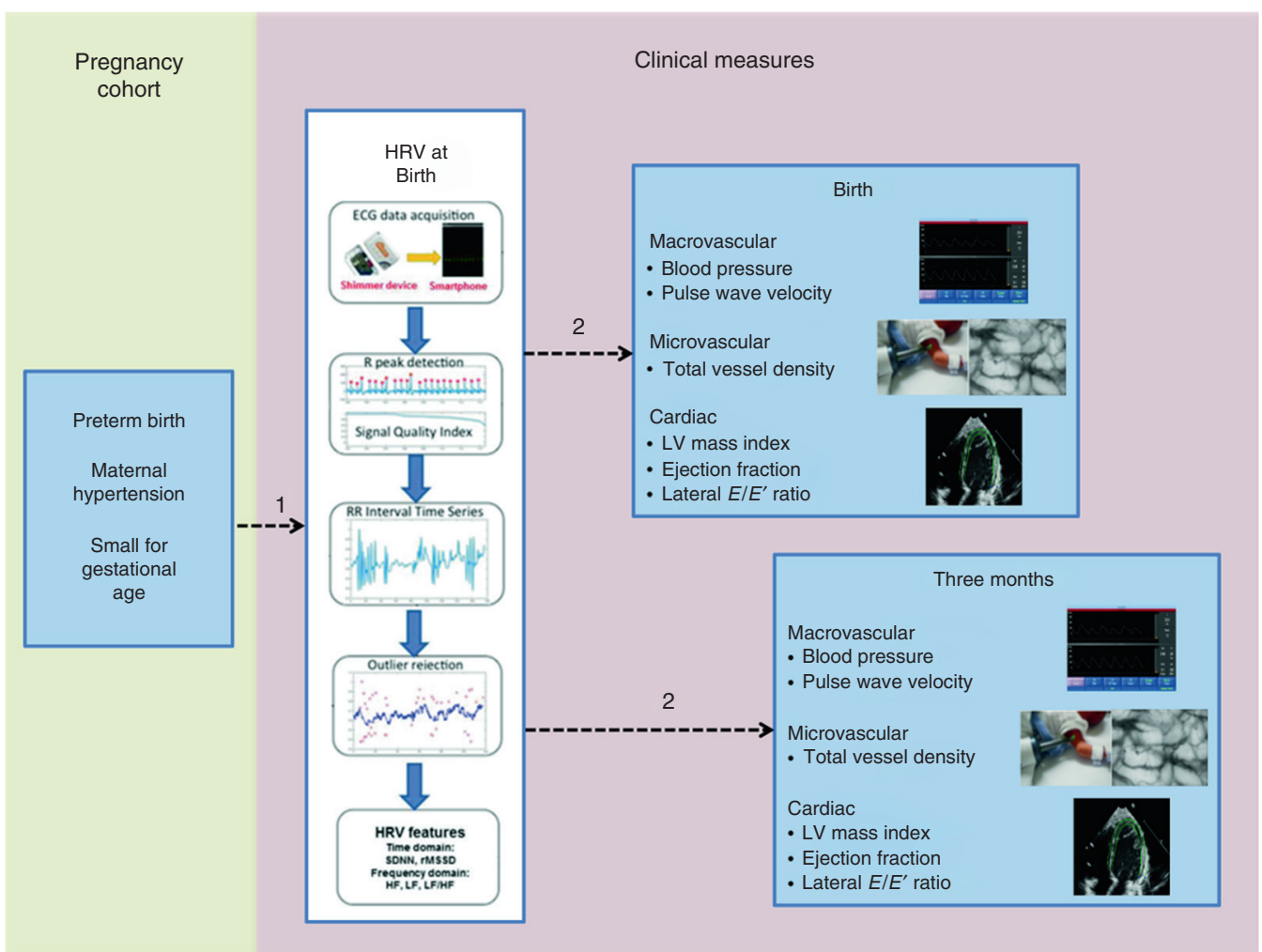

Fig. 1 Overview of study design investigating whether (1) pregnancy complications had an effect on heart rate variability at birth and (2) if heart rate variability at birth predicted cardiovascular development at birth or at 3 months of age, as measured by macrovascular, microvascular and cardiac assessments in the offspring. HRV heart rate variability, SDNN standard deviation of the NN intervals, rMSSD root mean square of the difference between adjacent NN intervals, HF high frequency, LF low frequency, LV left ventricular

datasets from birth and 3 months of age (Fig. 1) from participants in the EPOCH programme (Effect of Pregnancy on Offspring Cardiovascular Health study-approved by South Central Berkshire Research Ethics Committee ref. 11/SC/0006, UKCRN/clinical trials ref. NCT01888770).

All mothers gave written informed consent for the involvement of their children, in accordance with the Declaration of Helsinki, including permission to access maternal and offspring clinical records and link data between the studies. Mothers below the age of 16 years were excluded from the study as were those with chronic cardiovascular conditions prenatally, including hypertension. Infants were excluded if they had evidence of congenital cardiovascular disease (with the exception of Persistent Ductus Arteriosus and Atrial Septal Defect), chromosomal abnormalities or genetic disorders. Prolonged resuscitation at birth, intraventricular haemorrhage and ventilatory support during the time of assessment did not constitute the specific exclusion criteria, although no infants in our cohort fell into these categories.

\section{Clinical data collection and characterisation of pregnancy complications}

Characterisation of pregnancy complications and perinatal data related to the clinical care of the infant was extracted from the medical records and the questionnaires in a standardised way across studies by the same data collection team (C.A., E.D., Y.K.). Data collection details are available from https://clinicaltrials.gov (NCT01888770). The postmenstrual age at the time of measurements was calculated, relative to the gestational age defined at first trimester ultrasound. Hypertensive pregnancy (HTN) diagnosis (pregnancy-induced hypertension, pre-eclampsia (PET)) was defined according to ISSHP guidelines. ${ }^{14} Z$-scores for birthweight were calculated using the International Standard size at birth reference charts from the INTERGROWTH-21st Project ${ }^{15,16}$ using their online application (https://intergrowth21.tghn.org/globalperinatal-package/intergrowth-21st-comparison-application/). SGA was defined as a birthweight below the 10th centile.

\section{Cardiovascular measures}

Cardiovascular measurements were performed within 4 weeks of birth and again at 3 months of age in a temperature-controlled room, with the infant at rest, either in their mother's arms or in a crib. At both the birth and 3 month assessments, weight was measured using digital scales (Charder Model MS4200) to the nearest $0.01 \mathrm{~kg}$, with the infant fully naked. Head circumference was measured with a tape measure to nearest $\mathrm{mm}$. At birth and 3 months, three blood pressure measurements were recorded on the right calf with an automated digital monitor (Dinamap Technology ${ }^{\otimes}$ V100) using appropriate sized cuffs and were averaged for analysis. Brachial-femoral pulse wave velocity (PWV), in order to study arterial stiffness, was measured by fitting brachial and femoral cuffs a known distance apart using an oscillometry device (Vicorder, Skidmore Medical, Taunton, UK). Methods for echocardiography and in vivo microvascular imaging have been published previously and can be found in Supplemental Methods (online). ${ }^{10,17}$

Heart rate variability

Data acquisition. At birth assessment, all babies had a short 5-10 min ECG taken between feeds, lying down without the use of a pacifier. The Shimmer device was used to acquire the data, which was connected via Bluetooth to an Android mobile smartphone. The ECG collected consisted of two-lead signals with a $256 \mathrm{~Hz}$ sampling frequency and 12 bit quantization levels. Data were collected through an android app, ${ }^{18}$ processed and stored on the 
phone, before being transferred to a server. Details of whether the baby was asleep or awake were noted and the recording was stopped if there was excessive restlessness or crying.

Data processing. The ECG signals were then processed in order to extract the RR interval time series, but also a Signal Quality Index (SQI). These features were extracted using previously published techniques. ${ }^{19}$ The SQI was used in order to select the "best" 5-min segment, and the RR interval time series from this segment was kept for HRV analysis.

HRV analysis. The 5-min RR interval segment was used to extract HRV features. These features were extracted using the HRV toolkit, which has been validated and is freely available online. ${ }^{20}$ Processing included detection and extraction of the NN interval time series and automated outlier removal for rejection of artefactual RR points. The calculated HRV features were based on the basic time-domain HRV statistics used in the literature, specifically the standard deviation of the NN intervals (SDNN) and root mean square of the difference between adjacent NN intervals (rMSSD). The frequency-domain features were extracted using the Lomb periodogram, eliminating the need for evenly sampled data in contrast to the traditional Fast Fourier Transformation. The benefit of this is that sections of the recordings in which there are gaps or extreme noise in the data can be omitted. Parameters included the total spectral power of all NN intervals between 0.05 and $0.2 \mathrm{~Hz}$ (low-frequency power, LF), the total spectral power of all NN intervals between 0.2 and $1 \mathrm{~Hz}$ (high-frequency power, HF) and the ratio of low to high-frequency power (LF/HF ratio), using cut-offs previously suggested in the literature for the neonatal population. ${ }^{21}$ In order to be able to standardise these measures, only recordings during which the babies were asleep throughout were included in analysis.

Statistical analysis

Statistical analysis was performed using SPSS Version 22 (IBM, Armonk, NY) and GraphPad Prism 6.0 (La Jolla, CA). Comparison between the groups for continuous variables was carried out using a two-tailed, independent samples $t$-test for normally distributed variables and Mann-Whitney $U$ test for non-normally distributed data. Bivariate regression models were performed using a forced entry method and unstandardized B coefficients, and $95 \%$ confidence intervals are reported. The sample size, $n=$ 33 preterm and $n=65$ term offspring, provided us with $80 \%$ power at a significance level of $a=0.05$ to detect a difference of at least 0.85 standard deviation between the groups. $P$-values less than 0.05 were considered statistically significant.

\section{RESULTS}

Study population characteristics

The technology for assessment of HRV became available during the course of recruitment to the EPOCH study, and, therefore, out of the 266 infants in the full neonatal cohort, 140 infants had an ECG taken at birth. Of these, 3 were unanalysable due to poor signal quality and 39 were excluded, as the infant was awake or restless during acquisition. Maternal and offspring demographic and anthropometric characteristics in the cohort are presented in Table 1, with subgroup characteristics available in Supplemental Table S1 (online).

Pregnancy complications and HRV

Those born preterm had a higher heart rate and lower HRV than those born term (Fig. 2a). There was a positive association between SDNN, rMSSD, LF and HF, and gestational age at birth, although the association with SDNN failed to reach significance after adjusting for postnatal age at assessment and offspring sex (Table 2). There was a negative correlation between LF/HF ratio
Table 1. Cohort characteristics

\begin{tabular}{|c|c|}
\hline & $n=98$ \\
\hline \multicolumn{2}{|l|}{ Maternal demographics and anthropometrics } \\
\hline Maternal age at delivery, years & $33.0 \pm 4.6$ \\
\hline $\mathrm{BMI}$ at booking, $\mathrm{kg} / \mathrm{m}^{2}$ & $25.4 \pm 5.1$ \\
\hline Smokers, $n$ (\%) & $2(2)$ \\
\hline Maternal hypertension during pregnancy, $n(\%)$ & $46(47)$ \\
\hline \multicolumn{2}{|l|}{ Offspring birth characteristics } \\
\hline Gestational age at delivery, weeks & $37.9 \pm 2.9$ \\
\hline Males, $n(\%)$ & $48(49)$ \\
\hline Birth order ${ }^{a}$ & $1 \pm 1$ \\
\hline Caesarean section, $n$ (\%) & $36(37)$ \\
\hline Antenatal steroids, $n$ (\%) & $28(29)$ \\
\hline \multicolumn{2}{|l|}{ Offspring physiological measures at birth } \\
\hline Head circumference, $\mathrm{cms}$ & $33.5 \pm 2.2$ \\
\hline Birthweight, grams & $2964 \pm 754$ \\
\hline Birthweight Z-score & $0.10 \pm 1.0$ \\
\hline $\mathrm{sBP}, \mathrm{mmHg}$ & $81 \pm 15$ \\
\hline $\mathrm{dBP}, \mathrm{mmHg}$ & $44 \pm 10$ \\
\hline Pulse wave velocity $(\mathrm{m} / \mathrm{s})$ & $5.5 \pm 1.5$ \\
\hline \multicolumn{2}{|l|}{ Offspring physiological measures at 3 months } \\
\hline Weight, grams & $5763 \pm 950$ \\
\hline Head circumference, cms & $40.5 \pm 1.6$ \\
\hline $\mathrm{sBP}, \mathrm{mmHg}$ & $97 \pm 12$ \\
\hline $\mathrm{dBP}, \mathrm{mmHg}$ & $53 \pm 13$ \\
\hline Pulse wave velocity $(\mathrm{m} / \mathrm{s})$ & $6.5 \pm 1.7$ \\
\hline
\end{tabular}

and gestational age at birth even after adjustment. There was no significant difference in the heart rate or the HRV parameters between those born to mothers with or without maternal hypertension (Fig. $2 \mathrm{~b}$ and Table 2). We separately analysed those whose mothers had a more severe hypertensive disorder of pregnancy, classified as PET, and found no differences in the neonatal HRV in this group (Fig. 2c). Furthermore, there were no significant associations between birthweight $z$-score and any HRV parameter (Table 2) or any difference between those classified as small or appropriate for gestational age (Fig. 2d). Due to the small numbers of preterm babies in our cohort, we were not powered to detect differences between subgroups, for example, preterm SGA vs. preterm appropriate for gestational age. We therefore performed additional regression analyses to clarify the lack of relationship with pregnancy complications by studying the relationship between rMSSD at birth, correcting for postnatal age at assessment, offspring sex, as well as gestatinal age at birth (Supplemental Table S2 (online)). We found that even after adjusting for physiological changes in autonomic balance due to gestational age, there were still no correlations between other pregnancy complications with HRV.

We additionally studied whether particular perinatal clinical features including time on ventilation or APGAR score predicted autonomic dysfunction in those born preterm, but were not able to identify specific markers of more deranged function, except for a borderline association between caesarean delivery and a greater rMSSD (Table 3). When the subgroup analysis was performed on only babies that were born by caesarean section, there was no correlation between rMSSD and whether the delivery took place 

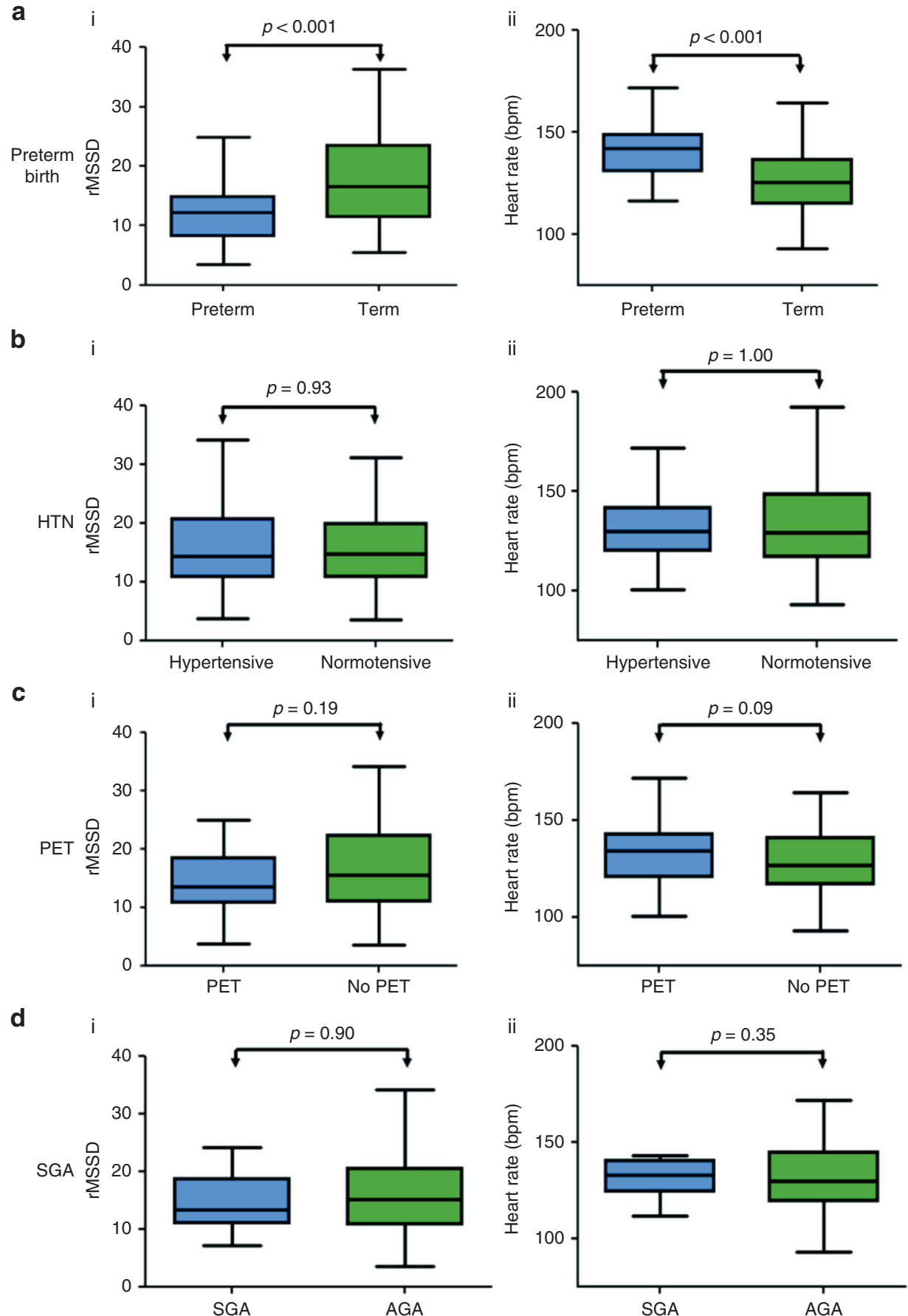

Fig. 2 Boxplots demonstrating a significantly decreased rMSSD and increased heart rate in the offspring born preterm (a), but no significant difference in those exposed to maternal hypertension (b), pre-eclampsia (c) or those born small for gestational age (d). rMSSD root mean square of the difference between adjacent NN intervals, HTN hypertensive pregnancy, PET pre-eclamptic pregnancy, SGA small for gestational age, AGA appropriate for gestational age

pre or during labour, after adjusting for offspring sex and postmenstrual age at assessment $(B=2.4095 \% \mathrm{Cl}(-3.74$ to 8.52), $p=0.43$ ).

Relationship with cardiovascular structure and function at birth and 3 months of age

We studied whether HRV was a predictor of other cardiovascular developmental differences at birth or related to those known to be found in preterm offspring, specifically postnatal cardiac hypertrophy and microvascular rarefaction. We based the analysis on associations with rMSSD at birth as this was the HRV parameter with the strongest association with gestational age. However, there was no association with these parameters (Table 4), even when the cohort was split into preterm and term groups (data not shown).

\section{DISCUSSION}

In this study, we have demonstrated that the HRV parameters derived from short-length ECG recordings in the first week of life 
Table 2. Multivariable regression coefficients for heart rate variability parameters at birth and pregnancy complications

\begin{tabular}{|c|c|c|c|c|c|c|c|c|c|}
\hline & \multicolumn{3}{|c|}{ Gestational age at birth (weeks) } & \multicolumn{3}{|c|}{ Maternal hypertension } & \multicolumn{3}{|c|}{ Birthweight $z$-score } \\
\hline SDNN & 0.76 & $-0.13-1.66$ & 0.09 & 2.30 & $-2.92-7.53$ & 0.38 & -1.92 & $-4.32-0.47$ & 0.11 \\
\hline rMSSD & 0.99 & $0.43-1.54$ & 0.001 & 0.24 & $-3.17-3.65$ & 0.89 & -0.41 & $-1.98-1.17$ & 0.61 \\
\hline LF & 23.50 & $6.02-40.98$ & 0.009 & 61.16 & $-42.84-165.16$ & 0.25 & -41.59 & $-89.25-6.07$ & 0.09 \\
\hline $\mathrm{HF}$ & 19.25 & $5.51-33.00$ & 0.007 & 9.28 & $-73.32-91.87$ & 0.82 & -10.85 & $-48.96-27.27$ & 0.57 \\
\hline
\end{tabular}

SDNN standard deviation of the NN intervals, rMSSD root mean square of the difference between adjacent NN intervals, LF low frequency, $H F$ high frequency $\mathrm{B}$ indicates unstandardized coefficient presented with $95 \% \mathrm{Cl}$ after correcting for postnatal age at assessment and offspring sex

Bold values are any $p$-values that are $<0.05$ i.e. significant

\begin{tabular}{|c|c|c|c|}
\hline & \multicolumn{3}{|l|}{ rMSSD } \\
\hline & $B$ & $95 \% \mathrm{Cl}$ & $p$-Value \\
\hline Maternal smoking & -3.29 & $-14.98-8.39$ & 0.58 \\
\hline Antenatal steroids & 0.11 & $-0.02-0.24$ & 0.58 \\
\hline Caesarean section & 3.63 & $-7.16--0.10$ & 0.04 \\
\hline Apgar score at $5 \mathrm{~min}$ & 0.20 & $-2.06-2.46$ & 0.86 \\
\hline Days of oxygen & 0.16 & $-1.04-1.36$ & 0.80 \\
\hline Postnatal infection & -1.28 & $-7.69-5.13$ & 0.69 \\
\hline \multicolumn{4}{|c|}{$\begin{array}{l}\text { rMSSD root mean square of the difference between adjacent NN intervals } \\
\mathrm{B} \text { indicates unstandardized coefficient presented with } 95 \% \mathrm{Cl} \text {, after } \\
\text { correcting for postmenstrual age at assessment (gestational age at birth } \\
\text { plus age at assessment) and offspring sex } \\
\text { Bold values are any } p \text {-values that are }<0.05 \text { i.e. significant }\end{array}$} \\
\hline
\end{tabular}

are significantly associated with gestational age at birth. HRV is decreased in preterm infants, compared to term counterparts, with reduced parasympathetic activity ${ }^{22}$ and a relative imbalance between sympathetic and parasympathetic tone, compared to term infants. ${ }^{23}$ In contrast, we found no association between HRV and exposure to maternal hypertension or fetal growth restriction within our cohort. We have also found no evidence that HRV at birth associates with patterns of cardiovascular development in the early postnatal period, which we have previously reported in those born preterm. ${ }^{10,17}$

Our findings are consistent with several previous studies that have also observed reduced HRV at birth in those born preterm. ${ }^{4}$ These changes could have been established in response to specific in utero stressors linked with the preterm birth. Alterations in maternal HRV are seen in pathological pregnancies, with PET having been associated with reduced maternal HRV, which worsens as the pregnancy progresses. ${ }^{3}$ Interestingly, there is evidence that this is of relevance to the child, as maternal autonomic heart rate modulation relates to fetal heart rate patterns in hypertensive pregnancies. ${ }^{3}$ However, our data suggests that any links between maternal and fetal heart rate do not persist after delivery in those born to hypertensive pregnancies, irrespective of the severity or classification of the hypertensive disorder.

Furthermore, even when there is evidence of fetal compromise, with a reduced birthweight $z$-score or classification as SGA, if there are any in utero differences in autonomic function, they are not evident after birth, although, interestingly, adults born SGA have been shown to have sympathetic nerve hyperactvity. ${ }^{24} \mathrm{~A}$ reason for this might be that those in our cohort born SGA were constitutionally small rather than being growth restricted, and were therefore unlikely to exhibit autonomic dysfunction. Data which would have differentiated between SGA and fetal growth restriction was unfortunately not available for our cohort. This was because babies were recruited postnatally and problems with growth were not suspected prior to birth in the majority of cases. Therefore, in utero measures of placental function had not been carried out by their clinical team. An alternative explanation for our, and others', findings in those born preterm is that they merely reflect a relative functional immaturity in the ANS activity. The fetal ANS develops progressively throughout pregnancy, ${ }^{25}$ with more rapid development of the parasympathetic branch, ${ }^{26}$ and therefore differences in the ANS function at birth would be expected, proportional to the gestational age.

Nevertheless, this functional immaturity at birth could still have pathological significance. Previous studies have demonstrated that deficits in HRV parameters in the preterm population may persist after birth up to term equivalent age, ${ }^{27}$ which suggests that normal development may require the foetus to remain in utero until term. ${ }^{7,26}$ Another potential hypothesis for this delayed or arrested maturation is disordered anatomical and cellular development of the nervous system ${ }^{28}$ or disruption of neuropeptide synthesis caused by inflammatory events, ${ }^{29}$ which are more common in the preterm population. HRV has been shown to be altered in conditions such as intraventricular haemorrhage, ${ }^{30}$ as well as being an indicator of the severity of clinical conditions such as respiratory distress syndrome ${ }^{6}$ and clinical illness scales. ${ }^{5}$ Nutritional, ${ }^{31}$ environmental ${ }^{32}$ or iatrogenic stress ${ }^{33}$ in the ex utero environment has also been potentially linked with abnormal ANS development. This may explain the borderline association between vaginal delivery and decreased HRV and vaginal delivery, as a caesarean section may mitigate some of the delivery stress accompanying complicated vaginal deliveries. However, there were no significant associations between rMSSD and Apgar score at 5 min (Table 3) and no correlation between HRV and whether the caesarean section was performed pre or during labour.

In our cohort, other perinatal exposures were not related to HRV, and HRV measures at birth were not predictive of the cardiac and vascular structure and functional changes that we have previously reported in preterm offspring in the postnatal period. Therefore, the altered ANS function is unlikely to explain these cardiovascular developmental differences in those born preterm. The lack of importance of the ANS function may be because our premature subgroup had an average gestational age of 34.4 weeks, and the frequency of severe clinical postnatal conditions was low. The ANS of late preterm infants matures more quickly after birth, ${ }^{34}$ and previous studies that showed continued reduction in HRV at term equivalent age have tended to be in the more extreme preterm infants born prior to 32 weeks. $^{35}$ Therefore, it remains possible that a functional immaturity of the ANS has a greater impact on cardiovascular development for the more extreme preterm infants. 
Table 4. Multivariable regression coefficients for cardiovascular development in early postnatal life and rMSSD at birth

\begin{tabular}{|c|c|c|c|c|c|c|}
\hline $\mathrm{sBP}(\mathrm{mmHg})$ & -0.11 & $-0.47-0.25$ & 0.55 & 0.07 & $-0.23-0.38$ & 0.63 \\
\hline $\mathrm{dBP}(\mathrm{mmHg})$ & -0.03 & $-0.27-0.20$ & 0.78 & -0.03 & $-0.34-0.28$ & 0.85 \\
\hline $\mathrm{PWV}(\mathrm{m} / \mathrm{s})$ & -0.01 & $-0.05-0.03$ & 0.63 & 0.01 & $-0.03-0.05$ & 0.60 \\
\hline \multicolumn{7}{|l|}{ Cardiac } \\
\hline Left ventricular mass index & 0.00 & $-0.09-0.09$ & 1.00 & -0.04 & $-0.18-0.12$ & 0.60 \\
\hline Ejection fraction (\%) & 0.06 & $-0.23-0.34$ & 0.69 & 0.20 & $-0.05-0.46$ & 0.11 \\
\hline Lateral $\mathrm{E} / \mathrm{E}^{\prime}$ ratio & 0.002 & $-0.07-0.07$ & 0.96 & -0.06 & $-0.11-0.01$ & 0.07 \\
\hline
\end{tabular}

An alternative explanation might be that HRV does not sufficiently assess the sympathetic function, in spite of it being a sensitive measure of overall autonomic imbalance, which allows for the evaluation of a proportion of cardiovagal function. Therefore, predominance of pathology in one of the ANS branches might be obscured by compensatory interactions with the other ANS branch not captured by HRV analysis. ${ }^{36}$ Since our data demonstrate that overall cardiac autonomic dysfunction relates to increasing prematurity, but appears not to associate with altered cardiovascular development, it clearly highlights the necessity of follow-up research to elucidate whether separate assessment of the sympathetic and the parasympathetic functional integrity (including non-cardiac measures) might provide additional insights into the mechanisms, whereby birth complications such as preterm birth affect the development of the cardiovascular system.

Whether the differences in the autonomic function that we observe at birth in those born preterm could be of relevance to the increased risk of hypertension in adulthood, independent of changes in cardiac and vascular phenotype, ${ }^{37}$ remain to be seen in future studies. HRV attenuation has long been implicated in adult cardiovascular disease states such as acute myocardial infarction ${ }^{1}$ and congestive heart failure. ${ }^{2}$ However, one longer-term study of preterm offspring HRV found that early differences were attenuated by 2 years of age and were equivalent to term-born offspring by 6-7 years of age, ${ }^{27}$ suggesting that there needs to be a re-emergence of HRV differences in adult life.

Studies of the ANS function in neonatal populations need to confront several challenges. We used state-of-the-art, small, remote monitoring technology to capture data, but our analysis needed to be based on short-length recordings. However, the other measures that we have derived from these short recordings have previously been shown to correlate well with the parameters measured from longer recordings in adults. ${ }^{38}$ Measurement of HRV is also only an indirect measurement of the autonomic function, but structural measures of the ANS such as skin nerve biopsies are not feasible in the neonatal population. In addition, specific measurements of the sympathetic function such as by measuring muscle sympathetic nerve activity by microneurography ${ }^{39}$ would be technically challenging in this age group, which might constitute one of the major challenges in future studies of the specific role of integrity of both ANS branches in cardiovascular development. We used the data on sleeping infants so as to control external conditions and stimulation to make it easier to interpret the differences in HRV parameters between groups of subjects. However, we did not differentiate between active and quiet sleep states using a simultaneous electroencephalogram, but, instead, stopped recordings during periods of observed unrest. Nevertheless, a previous study has suggested in healthy term neonates that there is no difference in HRV measures between the groups, when divided into behavioural states during sleep ${ }^{25}$ with close agreement between low mean heart rate and quiet sleep, and high mean heart rate and active sleep in infants. ${ }^{40}$ Mean respiration rate during the recording was also not recorded, although, again, a previous study has suggested that this may not correlate with HRV indices. ${ }^{25}$ In summary, HRV at birth is significantly associated with gestational age at birth with increasing prematurity resulting in increased differences in the autonomic function, compared to term infants, as suggested by reduced time and frequency domain the HRV parameters. No associations between HRV and maternal hypertension or fetal growth restriction were found. In addition, we found no evidence that the autonomic function at birth had an impact on cardiovascular development in the early postnatal period, but whether it in part explains the long-term risk of hypertension in offspring exposed to pregnancy complications remains to be seen.

\section{ACKNOWLEDGEMENTS}

We are grateful to all the pregnant women and babies who participated in this study. This work was supported by the British Heart Foundation (FS/11/65/28865) to Professor Leeson. Additional grants were received from the National Institute for Health Research Oxford Biomedical Research Centre and Oxford British Heart Foundation Centre for Research Excellence.

\section{ADDITIONAL INFORMATION}

The online version of this article (https://doi.org/10.1038/s41390-018-0021-0) contains supplementary material, which is available to authorized users.

Competing interests: The authors declare no competing interests.

Publisher's note: Springer Nature remains neutral with regard to jurisdictional claims in published maps and institutional affiliations.

\section{REFERENCES}

1. Lombardi, F. et al. Heart rate variability as an index of sympathovagal interaction after acute myocardial infarction. Am. J. Cardiol. 60, 1239-1245 (1987).

2. Casolo, G. et al. Twenty-four-hour spectral analysis of heart rate variability in congestive heart failure secondary to coronary artery disease. Am. J. Cardiol. 67, 1154-1158 (1991) 
3. Swansburg, M. L., Brown, C. A., Hains, S. M., Smith, G. N. \& Kisilevsky, B. S. Maternal cardiac autonomic function and fetal heart rate in preeclamptic compared to normotensive pregnancies. Can. J. Cardiovasc. Nurs. 15, 42-52 (2005).

4. Patural, $\mathrm{H}$. et al. Autonomic cardiac control of very preterm newborns: a prolonged dysfunction. Early Hum. Dev. 84, 681-687 (2008).

5. Goldstein, B. et al. Decomplexification in critical illness and injury: relationship between heart rate variability, severity of illness, and outcome. Crit. Care Med. 26, 352-357 (1998).

6. Prietsch, V., Knoepke, U. \& Obladen, M. Continuous monitoring of heart rate variability in preterm infants. Early Hum. Dev. 37, 117-131 (1994).

7. Spassov, L. et al. Heart rate and heart rate variability during sleep in small-forgestational age newborns. Pediatr. Res. 35, 500-505 (1994).

8. Lewandowski, A. J. et al. Elevated blood pressure in preterm-born offspring associates with a distinct antiangiogenic state and microvascular abnormalities in adult life. Hypertension 65, 607-614 (2015).

9. Lewandowski, A. J. et al. Preterm heart in adult life: cardiovascular magnetic resonance reveals distinct differences in left ventricular mass, geometry, and function. Circulation 127, 197-206 (2013).

10. $\mathrm{Yu}, \mathrm{G}$. Z. et al. Association of maternal antiangiogenic profile at birth with early postnatal loss of microvascular density in offspring of hypertensive pregnancies. Hypertension 68, 749-759 (2016).

11. Lewandowski, A. J. et al. Right ventricular systolic dysfunction in young adults born preterm. Circulation 128, 713-720 (2013).

12. Lazdam, M. et al. Unique blood pressure characteristics in mother and offspring after early onset preeclampsia. Hypertension 60, 1338-1345 (2012).

13. Davis, E. F. et al. Clinical cardiovascular risk during young adulthood in offspring of hypertensive pregnancies: insights from a 20-year prospective follow-up birth cohort. BMJ Open 5, e008136 (2015).

14. Brown, M. A., Lindheimer, M. D., de Swiet, M., Van Assche, A. \& Moutquin, J. M. The classification and diagnosis of the hypertensive disorders of pregnancy: statement from the International Society for the Study of Hypertension in Pregnancy (ISSHP). Hypertens. Pregnancy 20, IX-XIV (2001).

15. Villar, J. et al. Growth IFN2014 International standards for newborn weight, length, and head circumference by gestational age and sex: the newborn cross-sectional study of the INTERGROWTH-21st project. Lancet 384, 857-868 (2014).

16. Villar, J. et al. INTERGROWTH-21st Very preterm size at birth reference charts. Lancet 387, 844-845 (2016).

17. Aye, C. Y. L. et al. Disproportionate cardiac hypertrophy during early postnatal development in infants born preterm. Pediatr. Res. 82, 36-46 (2017).

18. Oster, J., Behar, J., Colloca, R., Li, Q., Clifford, G. D. Open source Java-based ECG analysis software and Android app for atrial fibrillation screening. Comput. Cardiol. 731-734 (2013).

19. Johnson, A. E., Behar, J., Andreotti, F., Clifford, G. D. \& Oster, J. Multimodal heart beat detection using signal quality indices. Physiol. Meas. 36, 1665 (2015).

20. Goldberger, A. L. et al. PhysioBank, PhysioToolkit, and PhysioNet: components of a new research resource for complex physiologic signals. Circulation 101 E215-E220 (2000).

21. Giddens, D. P. \& Kitney, R. I. Neonatal heart rate variability and its relation to respiration. J. Theor. Biol. 113, 759-780 (1985).

22. Pomeranz, B. et al. Assessment of autonomic function in humans by heart rate spectral analysis. Am. J. Physiol. 248, H151-H153 (1985).

23. Electrophysiology TFotESoCatNASoPa. Heart rate variability. Circulation 93 , 1043-1065 (1996).

24. Boguszewski, M. C., Johannsson, G., Fortes, L. C. \& Sverrisdottir, Y. B. Low birth size and final height predict high sympathetic nerve activity in adulthood. J. Hypertens. 22, 1157-1163 (2004).
25. Longin, E., Schaible, T., Lenz, T. \& Konig, S. Short term heart rate variability in healthy neonates: normative data and physiological observations. Early Hum. Dev. 81, 663-671 (2005).

26. Gagnon, R., Campbell, K., Hunse, C. \& Patrick, J. Patterns of human fetal heart rate accelerations from 26 weeks to term. Am. J. Obstet. Gynecol. 157, 743-748 (1987).

27. De Rogalski Landrot, l. et al. Autonomic nervous system activity in premature and full-term infants from theoretical term to 7 years. Auton. Neurosci. 136, 105-109 (2007).

28. Horner, S. M. et al. Contribution to heart rate variability by mechanoelectric feedback. Stretch of the sinoatrial node reduces heart rate variability. Circulation 94, 1762-1767 (1996).

29. Tracey, K. J. The inflammatory reflex. Nature 420, 853-859 (2002).

30. Watkins, T. W. et al. Heart rate variability: relationship to IVH in VLBW neonates (abstract). Pediatr. Res. 39, 251A (1996).

31. Lonsdale, D. Sudden infant death syndrome requires genetic predisposition some form of stress and marginal malnutrition. Med. Hypotheses 57, 382-386 (2001).

32. Goto, K. et al. More awakenings and heart rate variability during supine sleep in preterm infants. Pediatrics 103, 603-609 (1999).

33. Thiriez, G. et al. Heart rate variability in preterm infants and maternal smoking during pregnancy. Clin. Auton. Res. 19, 149-156 (2009).

34. Henslee, J. A., Schechtman, V. L., Lee, M. Y. \& Harper, R. M. Developmental patterns of heart rate and variability in prematurely-born infants with apnea of prematurity. Early Hum. Dev. 47, 35-50 (1997).

35. Scher, M. S. et al. Cardiorespiratory behavior during sleep in full-term and preterm neonates at comparable postconceptional term ages. Pediatr. Res. $\mathbf{3 6}$ 738-744 (1994).

36. Houle, M. S. \& Billman, G. E. Low-frequency component of the heart rate variability spectrum: a poor marker of sympathetic activity. Am. J. Physiol. 276 $\mathrm{H} 215-\mathrm{H} 223$ (1999).

37. de Jong, F., Monuteaux, M. C., van Elburg, R. M., Gillman, M. W. \& Belfort, M. B. Systematic review and meta-analysis of preterm birth and later systolic blood pressure. Hypertension 59, 226-234 (2012).

38. Bigger, J. T., Fleiss, J. L., Rolnitzky, L. M. \& Steinman, R. C. The ability of several short-term measures of $\mathrm{RR}$ variability to predict mortality after myocardial infarction. Circulation 88, 927-934 (1993).

39. Grassi, G. et al. Effects of amlodipine on sympathetic nerve traffic and baroreflex control of circulation in heart failure. Hypertension 33, 671-675 (1999).

40. Nugent, S. T. F. J. Spectral analysis of heart rate variability in children and young adults during sleep and awake states. Proc. 14th International Conf. IEEE/EMBS 2640-2641 (1992) Engineering in Medicine and Biology Society, 1992 14th Annual International Conference of the IEEE; doi: 10.1109/IEMBS.1992.5761639.

(i) Open Access This article is licensed under a Creative Commons Attribution 4.0 International License, which permits use, sharing, adaptation, distribution and reproduction in any medium or format, as long as you give appropriate credit to the original author(s) and the source, provide a link to the Creative Commons license, and indicate if changes were made. The images or other third party material in this article are included in the article's Creative Commons license, unless indicated otherwise in a credit line to the material. If material is not included in the article's Creative Commons license and your intended use is not permitted by statutory regulation or exceeds the permitted use, you will need to obtain permission directly from the copyright holder. To view a copy of this license, visit http://creativecommons. org/licenses/by/4.0/.

(c) The Author(s) 2018 